\title{
An algebraic view of structural induction
}

\author{
Claudio Hermida* Bart Jacobs**
}

\begin{abstract}
We propose a uniform, category-theoretic account of structural induction for inductively defined data types. The account is based on the understanding of inductively defined data types as initial algebras for certain kind of endofunctors $T: \mathbb{B} \rightarrow \mathbb{B}$ on a bicartesian/distributive category $\mathbb{B}$. Regarding a predicate logic as a fibration $p: \mathbb{P} \rightarrow \mathbb{B}$ over $\mathbb{B}$, we consider a logical predicate lifting of $T$ to the total category $\mathbb{P}$. Then, a predicate is inductive precisely when it carries an algebra structure for such lifted endofunctor. The validity of the induction principle is formulated by requiring that the 'truth' predicate functor $T: \mathbb{B} \rightarrow \mathbb{P}$ preserve initial algebras. We then show that when the fibration admits a comprehension principle, analogous to the one in set theory, it satisfies the induction principle. We also consider the appropriate extensions of the above formulation to deal with initiality (and induction) in arbitrary contexts, i.e. the 'stability' property of the induction principle.
\end{abstract}

\section{Introduction}

Inductively defined data types are understood categorically as initial algebras for 'polynomial' endofunctors $T: \mathbb{B} \rightarrow \mathbb{B}$ or a bicartesian/distributive category $\mathbb{B}$, as in [CS91, Jac95]. The category $\mathbb{B}$ is the semantic category in which types and (functional) programs are modelled, e.g. Cpo or Set.

We will show how initiality canonically endows such data types with induction principles to reason about them. Induction is a property of a logic over (the theory) $\mathbb{B}$.

Induction is a property of a logic over (the theory) $\mathbb{B}$. Categorically, such a logic corresponds to a fibration over $\mathbb{B}$, written as $\underset{\mathbb{B}}{\mathbb{P}}$. $\mathbb{P}$ is the category of 'predicates' and 'proofs', over the 'types' and 'terms' of $\mathbb{B}$. When $\underset{\mathbb{B}}{\mathbb{P}}$ is endowed with appropriate structure, intended to model certain logical connectives and quantifiers, $\mathbb{P}$ is bicartesian/distributive and $p$ preserves this structure. It is then possible to 'lift' the functor $T$ to an endofunctor $\operatorname{Pred}(T): \mathbb{P} \rightarrow \mathbb{P}$ over $T$, i.e. $p \operatorname{Pred}(T)=T p$. The key point is that, given a $T$-algebra $T X \stackrel{x}{\longrightarrow} X$ and a predicate $P$ on $X$, i.e. $p P=X, P$ is inductive, meaning that it satifies the premise of the structural induction principle for the "type structure' $T$, precisely when it has a $\operatorname{Pred}(T)$-algebra structure $\operatorname{Pred}(T) P \stackrel{\bar{x}}{\longrightarrow} P$ with $p \bar{x}=x$. This observation leads to our definition of the induction principle relative to the fibration $p$ as the preservation of initial algebras by the 'truth predicate' functor $T: \mathbb{B} \rightarrow \mathbb{P}$, which assigns to the object (or 'type') $X$ the 'constantly true' predicate $T_{X}$.

As for the usual induction principle for the natural numbers $\omega$ in Set, we know it is valid using the initiality of $\omega$ with respect to the inductive subset $\{x \in X \mid P(x)\}$, determined by the inductive predicate $P$ which we wish to prove. This argument depends crucially on the fact that we can perform comprehension. In categorical terms, comprehension $P \mapsto\{x \in X \mid P(x)\}$ amounts to a right adjoint to $T: \mathbb{B} \rightarrow \mathbb{P}$, after [Law70].

* Computer Science Department, Aarhus University, DK-8000 Denmark.

e-mail: chermidaedaimi.aau.dk. The author acknowledges funding from the CLICS II ESPRIT project.

** CWI, Kruislaan 413, 1098 SJ Amsterdam, The Netherlands. e-mail: bjacobs@cri.nI 
With our abstract formulation of induction, we will show that when $\underset{\mathbb{B}}{\mathbb{P}}$ admits comprehension in the above sense, the induction principle holds in $p$, analogously to the above situation in Set.

This last fact that comprehension entails induction hinges on the fact that adjunctions between $\mathbb{B}$ and $\mathbb{P}$ induce adjunctions between the associated categories of algebras, $T-\mathcal{A l} g$ and $\operatorname{Pred}(T)-\mathcal{A l g}$ respectively, assuming some appropriate additional structure. This is a 2-categorical property, namely the 2 -functoriality of (the construction of) inserters: $T$ - $\mathcal{A l g}$ is the inserter of $T, 1_{\mathbb{B}}: \mathbb{B} \rightarrow \mathbb{B}$, in the sense of [Kel89]. See Theorems 2.3.1 and 4.0 .8 below.

Another important aspect of the present work is the consideration of the (frequently ignored) 'stability' of the induction principle under context weakening. This means that we should be able to reason by induction on a given data type not only when such type is given on its own, but also when it occurs toghether with some other data, which in turn may be subject to certain hypotheses. Technically, this amounts to the requirement that initiality of algebras be preserved under addition of indeterminates.

The primary aim of this work is to give a technically precise categorical formulation of a logical principle, namely structural induction. Such formulation makes the principle amenable to a purely algebraic manipulation. There are several relevant references in the literature, particularly [LS81, Pit93]. We would like to emphasise the following points, which highlight differences between our work and these references:

(i) The understanding of a predicate logic as a fibration is central to the present work. This provides not only an appropriate level of generality but also the right technical framework. In particular, the relationship between inductive predicates and logical predicates is best presented in this setting, as logical predicates for type constructors given by adjoints arise uniformly from an intrinsic property of adjunctions between fibrations, $c f$. [Her93].

(ii) The categorical framework which we work in takes explicit account of proofs of entailments between predicates. Thus this work can be seen as a generalisation of induction principles from the usual proof-irrelevant setting to the type-theoretic (or constructive) one. See Remark 2.2 .1 below.

(iii) 2-categorical reasoning is essential to get conceptually uniform formulations. For instance, just as inductive datatypes are understood as initial algebras for an endomorphism in Cat, the 2-category of small categories, their associated induction principles are formulated in terms of (distinguished) initial algebras for endomorphisms in $\mathrm{Cat} \rightarrow$. Similarly for stability of data types and their associated induction principles under context weakening: the former means preservation of initial algebras by addition of indeterminates in Cat while the latter amounts to the same kind of preservation in $\mathcal{F} i b$, the 2-category of fibrations. See $\$ 5$ below.

Background material on fibrations can be found in [Jac91, Pav90]. Indeterminates for fibrations, as relevant to this work, are discussed in [HJ93]. Inserters are presented in [Kel89]; they play a purely technical role here and hence they are not essential to understand the paper.

The material presented here is essentially an extension of [Her93, §4.5], combined with [Jac95]. A follow-up in [HJ95] deals with a dual coinduction principle (which holds in the presence of quotients) and a mixed induction/coinduction principle for mixed variance type-constructors, $c f$. [Pit93]. 


\section{Setting}

In this section we lay down the setting required for our formulation of structural induction. In $\S 2.1$ we define the kind of endofunctors whose initial algebras are understood as inductively defined datatypes and recall how such initial algebras may be obtained under suitable cocompleteness conditions. In $\$ 2.2$ we present the basic properties on fibrations required to give a categorical counterpart of a logic suitable to describe structural induction, including the description of logical products and logical coproducts.

\subsection{Inductive data types in a bicartesian category}

Following [CS91, Jac95], we will consider inductive data types in a bicartesian category $\mathbb{B}$, i.e. a category with finite products and coproducts. Actually, these references consider an additional of distributivity, but it is irrelevant until we consider 'stability', that is the preservation of initial algebras by weakening to arbitrary contexts, in $\S 5$.

We write $!_{A}: A \rightarrow 1$ for the unique morphism into the terminal object 1 and

$$
A \stackrel{\pi_{A, B}}{\stackrel{\pi^{\prime}}{\leftrightarrow}} A \times B \stackrel{{ }_{A, B}}{\longrightarrow} B
$$

for a product diagram in $\mathbb{B}$, omitting subscripts whenever convenient. Dually, we write

$$
A \stackrel{\iota_{A, B}}{\longrightarrow} A+B \stackrel{\iota_{A, B}^{\prime}}{\stackrel{1}{\longrightarrow}} B
$$

for a coproduct diagram.

Clearly, categories like $\mathcal{S} e t$ and $\mathcal{C} p o$ (with strict continuous functions) are bicartesian.

2.1.1. Inductive data types in a bicartesian category are specified by endofunctors, which give the 'signature' of the type. Given an endofunctor $T: \mathbb{B} \rightarrow \mathbb{B}$, we write $T$-Alg for the category whose objects, called $T$-algebras, are pairs $(X, x: T X \rightarrow X)$ and whose morphisms $f:(X, x) \rightarrow(Y, y)$ are $f: X \rightarrow Y$ in $\mathbb{B}$ such that $f \circ x=y \circ T f$. Ins $\left(T, 1_{\mathbb{B}}\right)$

2.1.2. Definition. Let $\mathbb{B}$ be a distributive category, $S$ a finite set and $M: S \rightarrow \mathbb{B}$ be a functor, regarding $S$ as a discrete category.

(i) Let $\mathcal{T}_{M} \subseteq|\mathrm{Cat}(\mathbb{B}, \mathbb{B})|$ be the least set of endofunctors on $\mathbb{B}$ such that

- The identity functor is in $\mathcal{T}_{M}$.

- For any $I \in S$, the constant functor $X \mapsto M(I)$, written $K_{M(I)}$ is in $\mathcal{T}_{M}$.

- The constants functors $K_{0}$ and $K_{1}$ are in $\mathcal{T}_{M}$.

- If $T_{1}$ and $T_{2}$ are in $T_{M}$, so are $T_{1} \times T_{2}$ and $T_{1}+T_{2}$, i.e. $\left(X \mapsto T_{1}(X) \times T_{2}(X)\right)$ and $\left(X \mapsto T_{1}(X)+T_{2}(X)\right)$ respectively. These operations are the product/coproduct in the functor category $\operatorname{Cat}(\mathbb{B}, \mathbb{B})$.

(ii) An inductive data type specification in $\mathbb{B}$ is given by a functor $M: S \rightarrow \mathbb{B}$ and a functor $(T: \mathbb{B} \rightarrow \mathbb{B}) \in \mathcal{T}_{M}$. We write $T_{M}$ for such specification and refer to it simply as a polynomial functor.

(iii) A model for a $T_{M}$ is a $T_{M}$-algebra.

(iv) The initial model for a specification $T_{M}$ is the initial $T$-algebra.

The set $S$ in the above definition is called a parameter set. Its role is to specify, via the functor $M: S \rightarrow \mathbb{B}$, those objects of $\mathbb{B}$ which are parameters for the data type specified. The examples below will make this clear. See [Jac95] for a more general typetheoretic formulation of data types in distributive categories. The initial $T$-algebra of a 
functor $T: \mathbb{B} \rightarrow \mathbb{B}$ need not exist. But it is possible to guarantee the existence of initial $T$-algebras under suitable cocompleteness conditions on $\mathbb{B}$ and $T$. As shown in [LS81], an initial $T$-algebra can be obtained as the colimit of an $\omega$-chain, when $T$ preserves such colimits. An $\omega$-chain is a functor $\omega \rightarrow \mathbb{B}$, where $\omega$ is the poset category of natural numbers with their usual ordering. The initial $T$-algebra is the colimit of the following $\omega$-chain:

$$
0 \stackrel{\iota}{\longrightarrow} \mathrm{TO} \stackrel{T_{\iota}}{\longrightarrow} T^{2} 0 \quad \ldots
$$

where $\iota: 0 \rightarrow T 0$ is the unique morphism from the initial object. In $\mathcal{S}$ et and $\mathcal{C} p o$, any

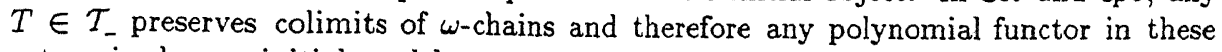
categories has an initial model.

2.1.3. An important observation due to Lambek, cf. [LS81] for instance, is that for an initial $T$-algebra $(D$, constr : $T D \rightarrow D)$, constr is an isomorphism. Thus, we can regard $D$ as the 'least fixed point' of $T$, as illustrated by the above $\omega$-chain. The isomorphism constr provides the 'constructors' of the data type, as the following familiar examples illustrate.

\subsubsection{EXamples. Let $\mathbb{B}$ be a bicartesian category.}

(i) Natural numbers object: Consider the polynomial functor $T X=1+X$, with parameter set $\emptyset$. A $T$-algebra $(A,[c, f]: T A \rightarrow A)$ is given by an object $A$, the 'carrier' of the type, and morphisms $c: 1 \rightarrow A$ and $f: A \rightarrow A$. An initial model for $T$ is precisely a natural numbers object $(N,[z, s])$ in Lawvere's sense, see [LS86, Part I, $\$ 9]$. In Set, it is the set of natural numbers $\omega$, with the usual zero and successor operations. Initiality means that there is an 'iterator', which given $c$ and $f$ as above produces a unique morphism $h: \mathrm{N} \rightarrow A$ such that $h \circ z=c$ and $h \circ s=f \circ h$. In Set, $h$ corresponds to the function defined from $c$ and $f$ by primitive recursion, given by $n \mapsto f^{(n)}(c)$. We write it $(c, f)$ for $h$ above.

(ii) Lists: For an object $A \in|\mathbb{B}|$, consider the polynomial functor $T_{A} X=1+A \times X$, for a singleton parameter set, i.e. $A:\{*\} \rightarrow \mathbb{B}$. A $T$-algebra is given by an object $B$ and morphisms $c: 1 \rightarrow B$ and $t: A \times B \rightarrow B$. An initial model in Set is precisely the set List $(A)$ of finite lists of elements of $A$, with the usual operations nil : $1 \rightarrow$ List $(A)$, the empty list, and cons : $A \times \operatorname{List}(A) \rightarrow \operatorname{List}(A)$, which given $a \in A$ and a list $l$, returns this list with the element $a$ appended to its head.

The example of lists above shows the role of the parameter $S$ and the functor $M: S \rightarrow \mathbb{B}$ in the specification of a data type; the type of lists List $(A)$ is parameterised by the type $A$ of the elements of the list.

\subsection{Logic over a bicartesian category}

Given a bicartesian category $\mathbb{B}$ in which we model inductive datatypes, we want a categorical formulation of a logic over it, a predicate logic over the 'types' and 'terms' of $\mathbb{B}$, in order to consider induction principles. The proper categorical version of a predicate logic over a category is embodied by the notion of fibration. We refer to [Jac91, Pav90] for an exposition of this point of view.

Thus a predicate logic corresponds to a fibration over $\mathbb{B}$, written as $\underset{\mathbb{P}}{\mathbb{P}} . \mathbb{P}$ is the category of 'predicates' and 'proofs', over the 'types' and 'terms' of $\mathbb{B}$. This can be made precise via the internal language of a fibration, in the same vein as a cartesian closed category has associated a simply typed $\lambda$-calculus as its internal language, $c f$. [LS86].

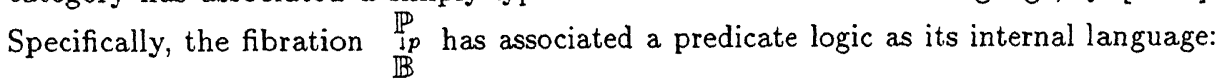


regarding $\mathbb{B}$ as a simple type theory, with product and coproduct types (see [Jac95]), an object $P$ of $\mathbb{P}$, with $p P=X$ is construed as a predicate, or indexed proposition, on the type $X$ :

$$
x: X \vdash P(x) \text { Prop }
$$

where we have written $P(x)$ to emphasize the dependency on the variable $x$, although we will usually leave this implicit. A morphism $h: P \rightarrow Q$ with $p h=u: X \rightarrow Y$, corresponds to a (unique) vertical morphism $\hat{h}: P \rightarrow u^{*}(Q)$, where $u^{*}(Q)$ is the domain of a cartesian lifting of $u$ at $Y$. In the predicate logic of $p$, this vertical morphism $\hat{h}$ corresponds to a proof of the entailment

$$
x: X \mid a: P(x) \vdash \hat{h}: Q(u(x))
$$

where $Q(u)$ is the predicate corresponding to $u^{*}(Q)$; reindexing in the fibration corresponds to substitution in the logic:

$$
(x: X \vdash u(x): Y, y: Y \vdash Q(y) \text { Prop }) \stackrel{u^{*}}{\longrightarrow} x: X \vdash Q(u(x)) \text { Prop }
$$

2.2.1. Remark. Although we usually omit the 'proof term' $\hat{h}$ in entailments, the reader should bear in mind that our approach is truly constructive, i.e. takes proofs into account.

2.2.2. . Fibrations are organized into the 2-category $\mathcal{F} i b$, whose objects are fibrations $\underset{\mathbb{B}}{\mathbb{E}} \underset{\mathbb{B}}{\mathbb{E}}$. Morphisms are given by commuting squares

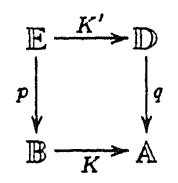

where $K^{\prime}$ preserves cartesian morphisms. Given morphisms $\left(K^{\prime}, K\right),\left(L^{\prime}, L\right): p \rightarrow q$, a 2 cell from $\left(K^{\prime}, K\right)$ to $\left(L^{\prime}, L\right)$ is a pair of natural transformations $\left(\sigma^{\prime}: K^{\prime} \Rightarrow L^{\prime}, \sigma: K \Rightarrow L\right)$ with $\sigma^{\prime}$ over $\sigma$, i.e. $q \sigma^{\prime}=\sigma p$.

$\mathcal{F}_{i} b$ is a sub-2-category of $\mathrm{Cat} \rightarrow$, whose objects are arbitrary functors $p, q, \ldots$ and whose morphisms are commuting squares as above (without any preservation properties).

The analysis of structural induction in $\S 3$ below, depends crucially on the relationship between the 'logical' structure of the fibration $\underset{1 p}{\mathbb{P}}$ and the categorical structure of the 'total' category $\mathbb{P}$. Specifically, we want to lift an endofunctor $T: \mathbb{B} \rightarrow \mathbb{B}$, belonging to $\mathcal{T}_{M}$, to one on $\mathbb{P}$. Since the functors on $\mathcal{T}_{M}$ are essentially those expressible by the bicartesian structure of $\mathbb{B}$, we need the same structure on $\mathbb{P}$. This leads us to consider the following kind of fibrations.

2.2.3. Definition. A bicartesian fibration $\underset{\mathbb{P}}{\mathbb{P}}$ is a fibration over a bicartesian category $\mathbb{B}$, such that $\mathbb{P}$ is bicartesian and $p$ strictly preserves such structure.

2.2.4. REMARK. A bicartesian fibration is a bicartesian object in $\mathcal{F} i b$, the 2-category of fibrations described in 2.2 .2 above. See [Her93] and the references there for details on such matters.

\footnotetext{
We assume a choice of cartesian liftings, i.e. we assume the fibration is cloven. Such a choice is always possible if we appeal to the axiom of choice.
} 
2.2.5. Examples. (i) Classical logic. The fibration corresponding to classical first-

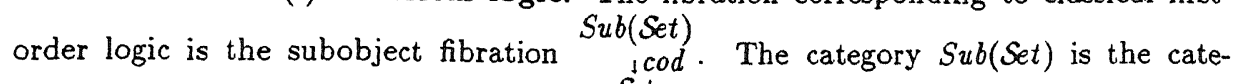
gory of subobjects: its objects are pairs $(S, X)$, where $S \subseteq X$, and its morphisms $f:(S, X) \rightarrow\left(S^{\prime}, X^{\prime}\right)$ are functions $f: X \rightarrow X^{\prime}$ such that $f(S) \subseteq S^{\prime}$. The fibration simply 'forgets' the subsets. Cartesian liftings are given by inverse images:

$$
\left(f: X \rightarrow X^{\prime},\left(S^{\prime}, X^{\prime}\right)\right) \mapsto\left(f^{-1}\left(S^{\prime}\right), X\right)
$$

The bicartesian structure of $\operatorname{Sub}(\operatorname{Set})$ is described below, in terms of logical predicates.

(ii) Admissible subsets. A related example is the fibration $\begin{gathered}A S u b\left(C_{p o}\right) \\ 1 \\ \mathcal{C}_{p o} \\ c_{0}\end{gathered}$, where $A S u b\left(C_{p o}\right)$ is the category of admissible subsets: its objects are pairs $(S, C)$ where $C$ is an $\omega$-cpo and $S \subseteq C$ is a subset containing the bottom element and closed under lub's of $\omega$-chains, while its morphisms are the strict continuous functions which respect the subsets, as in the preceeding example. The category $A S u b\left(C_{p o}\right)$ is bicartesian as it is a reflective subcategory of the fibred category $U^{*}(\operatorname{Sub}(\mathcal{S e t}))$, obtained from the 'classical logic' fibration by change-of-base along the forgetful functor $U: \mathcal{C} p o \rightarrow \operatorname{Set}$. See [Her93, $\$ 4.3 .2]$ for further details.

\subsubsection{Logical predicates}

In order to convey the logical significance of the bicartesian structure of $\mathbb{P}$ we recall, from [Her93], how such finite products and coproducts are induced by the fibred ones and the ones in the base.

\subsubsection{Proposition. Given $\underset{\mathbb{B}}{\mathbb{P}}$ with}

- $\mathbb{B}$ a bicartesian category,

- $p$ a fibred bicartesian category, i.e. every fibre is a bicartesian category and reindexing functors preserve finite products and coproducts,

- $p$ has coreindexing functors along coproduct injections, $I \stackrel{\iota}{\rightarrow} I+J \stackrel{\iota^{\prime}}{\leftarrow} J$, for every $I, J \in|\mathbb{B}|$.

Then, $\mathbb{P}$ is a bicartesian category and $p$ strictly preserves finite products and coproducts.

Proof. Given objects $P$ and $Q$ of $\mathbb{P}$, with $p P=I$ and $p Y=J$, their product in $\mathbb{P}$ is

$$
P \stackrel{\overline{\pi_{I, J}}}{\longleftarrow} \pi_{I, J}^{*}(P) \stackrel{\pi}{\longleftarrow}\left(\pi_{I, J}^{*}(P) \times_{I \times J}\left(\pi_{I, J}^{\prime}\right)^{*}(Q)\right) \stackrel{\pi^{\prime}}{\longrightarrow}\left(\pi_{I, J}^{\prime}\right)^{*}(Q) \stackrel{\overline{\pi_{I, J}^{\prime}}}{\longrightarrow} Q
$$

over the product $I \stackrel{\pi_{I, J}}{\leftarrow} I \times J \stackrel{\pi_{I, J}^{\prime}}{\rightarrow} J$, where $\times_{I \times J}$ is the product in the fibre $\mathbb{P}_{I \times J}$. Dually, their coproduct is

$$
P \stackrel{\iota_{I, J}}{\longrightarrow}\left(\iota_{I, J}\right) !(P) \stackrel{\iota}{\longrightarrow}\left(\iota_{I, J}\right) !(P)+_{I+J}\left(\iota_{I, J}^{\prime}\right):(Q) \stackrel{\iota^{\prime}}{\longleftarrow}\left(\iota_{I, J}^{\prime}\right) !(Q) \stackrel{\iota_{I, J}^{\prime}}{=} Q
$$

where $\underline{\iota_{I, J}^{\prime}}$ is a cocartesian lifting. Terminal and initial objects are obtained similarly $\square$ 
2.2.7. REMARK. In the internal language of $p$, the above construction of products reads as follows: given $x: I \vdash P$ Prop and $y: J \vdash Q$ Prop, their logical product is

$$
x: I, y: J \vdash P(x) \wedge Q(y) \text { Prop }
$$

and their logical coproduct is

$$
z: I+J \vdash(\exists x: I . \iota(x)=z \wedge P(x)) \vee\left(\exists y: J . \iota^{\prime}(y)=z \wedge Q(y)\right) \text { Prop }
$$

that is, a predicate over $I+J$ defined 'by cases'. This last expression of coproducts relies on the presence of an equality predicate, satisfying certain exactness conditions, commonly satisfied (see [Law70]). Actually, such additional structure on a fibration is irrelevant for our arguments; the above description is given only to emphasise the logical significance of the coproduct in $\mathbb{P}$. The relationship between categorical structure on $\mathbb{P}$ and logical predicates is further analysed in [Her93].

For example, the fibration $\begin{gathered}S u b(\text { Set }) \\ 1 \text { cod } \\ \text { Set }\end{gathered}$ satisfies the hypothesis of Proposition 2.2.6. The fibred products and coproducts are given by intersection and union, respectively. It has cocartesian liftings along arbitrary morphisms: given $S \subseteq X$ and $f: X \rightarrow X^{\prime}$, the lifting is the direct image $f(S) \subseteq X^{\prime}$.

\subsection{Adjunctions between categories of algebras}

We present the main technical tool we need to deal with adjunctions between categories of algebras, induced by adjunctions between the base categories. The result holds in any 2-category which admits inserters, as categories of algebras are an instance of such limits $c f$. [CS91], i.e. for a given $T: \mathbb{A} \rightarrow \mathbb{A}, T-\mathcal{A l g} \simeq \operatorname{Ins}\left(T, 1_{\mathbb{A}}\right)$, the inserter of $T$ and the identity functor on $\mathbb{A}$.

2.3.1. Theorem. Given a diagram in a 2-category $\mathcal{K}$

$$
\left.\stackrel{f}{\forall \stackrel{t}{\longrightarrow}} \stackrel{\alpha}{\Rightarrow}\right|_{B} ^{\longrightarrow} f
$$

in which $\alpha$ is an isomorphism and $f$ has a right adjoint, $\eta, \epsilon: f \dashv g$, the adjoint mate of $\alpha^{-1}$, i.e. $\theta=g t^{\prime} \in \circ g^{\prime} \alpha^{-1} g \circ \eta t g: t g \Rightarrow g t^{\prime}$, induces a morphism $g-\mathcal{A l g}: t^{\prime}-\mathcal{A l g} \rightarrow t-\mathcal{A l g}$

$$
\left.(B, t B \stackrel{a}{\longrightarrow} B) \quad \mapsto \quad\left(g B, t^{\prime}(g B) \stackrel{\alpha_{B}}{\longrightarrow} g t B \stackrel{g a}{\longrightarrow} g B\right)\right)
$$

right adjoint to the morphism $f-\mathcal{A l g}: t-\mathcal{A l g} \rightarrow t^{\prime}-\mathcal{A l g}$ induced by the above diagram, i.e.

$$
\left.(A, t A \stackrel{a}{\longrightarrow} A) \quad \mapsto \quad\left(f A, t^{\prime}(f A) \stackrel{\alpha_{A}}{\longrightarrow} f t A \stackrel{f a}{\longrightarrow} f A\right)\right)
$$

\section{Induction principle for inductive data types relative to a fibra- tion}

3.0.2. Given a set of parameters $S$ and functors $M: S \rightarrow \mathbb{B}$ and $\widetilde{M}: S \rightarrow \mathbb{P}$ such that $p \widetilde{M}=M$, a polynomial functor $T_{M}: \mathbb{B} \rightarrow \mathbb{B}$ induces a polynomial functor $\operatorname{Pred}(T)_{\tilde{M}}: \mathbb{P} \rightarrow \mathbb{P}$ 
fibred over $T$, using the bicartesian structure of $\mathbb{P}$. The formal definition of $\operatorname{Pred}(T)_{\tilde{M}}$ proceeds by induction on the construction of $T \in \mathcal{T}_{M}$. For instance, given $P \in\left|\mathbb{P}_{A}^{M}\right|$, $T_{A} X=1+A \times X$ induces $\operatorname{Pred}(T) Y=\tilde{1} \tilde{+} P \tilde{\times} Y$. We can then consider $\operatorname{Pred}(T)$-algebras and initial models in $\mathbb{P}$. We call $\operatorname{Pred}(T)_{\tilde{M}}$ the logical-predicate lifting of $T_{M}$. We thus get the following endomorphism in $\mathrm{Cat}^{\rightarrow}$

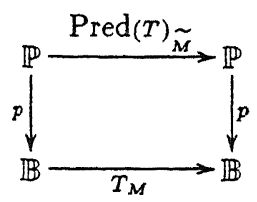

In a bicartesian fibration, the fibred terminal object (the 'truth predicate') is given by a functor $T: \mathbb{B} \rightarrow \mathbb{P}$, which is a (fibred) right adjoint to $p: \mathbb{P} \rightarrow \mathbb{B}$. Such a fibred terminal object is used to give a notion of provability in the 'logic' $p$. A 'predicate' $P$, with $p P=I$ is provable when there exists a morphism $h: \top_{I} \rightarrow P$ in the fibre $\mathbb{P}_{I}$. In the internal language of $p, c f . \S 2.2$, this amounts to a proof of the entailment

$$
x: I \mid a: \mathrm{T}_{I} \vdash h: P(x)
$$

We usually omit $a: T_{I}$ on the left-hand side of a sequent.

3.0.3. Given a polynomial functor $T_{M}$ in $\mathbb{B}$, with $M: S \rightarrow \mathbb{B}$ as in Def.2.1.2, we can consider the logical predicate lifting of $T$, using the functor $T M: S \rightarrow \mathbb{P}$. We write $\operatorname{Pred}(T): \mathbb{P} \rightarrow \mathbb{P}$ for the functor $\operatorname{Pred}(T)_{T M}$ so obtained. We thus have an endomorphism $(\operatorname{Pred}(T), T): p \rightarrow p$ in $C_{a t} \rightarrow$, and, writing $(1,1)$ for the identity on $p$, we can consider the inserter $\operatorname{Ins}((\operatorname{Pred}(T), T),(1,1))$ in $C a t \rightarrow$. We write $p-\mathcal{A} l g: \operatorname{Pred}(T)-\mathcal{A l} l g \rightarrow T-\mathcal{A l} g$ for the fibration so obtained. Here, $\operatorname{Pred}(T)-\mathcal{A l g}$ is the category of $\operatorname{Pred}(T)$-algebras of the endofunctor $\operatorname{Pred}(T)$ on $\mathbb{P}$, in agreement with our convention in 2.1.1. Furthermore, the adjunction $p, T: \mathbb{B} \dashv \mathbb{P}$ induces an adjunction $p-\mathcal{A} l g \dashv T$-Alg $: T-\mathcal{A} l g \rightarrow \operatorname{Pred}(T)-\mathcal{A l} g$,

by Theorem 2.3.1. In elementary terms, the functor $T$-Alg $: T$-Alg $\rightarrow \operatorname{Pred}(T)-\mathcal{A l} g$ acts as follows

$$
(I, T I \stackrel{i}{\longrightarrow} I) \quad \mapsto \quad\left(T_{I}, \operatorname{Pred}(T) T_{I} \stackrel{!}{\longrightarrow} T_{T I} \stackrel{T(i)}{\longrightarrow} T_{I}\right)
$$

using the fact that $T_{I}$ is terminal in the fibre $\mathbb{P}_{I}$.

Since the functor $p$-Alg $: \operatorname{Pred}(T)-\mathcal{A l g} \rightarrow T$ - $\mathcal{A l g}$ has a right adjoint, it preserves initial algebras. Hence, if $\operatorname{Pred}(T)-\mathcal{A l g}$ has an initial algebra, we may assume it lies over the initial algebra in $T$-Alg.

We are now in position to state our main definition.

3.0.4. DEFINITION (Induction principle in a fibration). Let $\underset{\mathbb{B}}{\mathbb{P}}$ be a bicartesian fibration, and let $T_{M}: \mathbb{B} \rightarrow \mathbb{B}$ be a polynomial functor, for a parameter set $S$ and a functor $M: S \rightarrow \mathbb{B}$. The fibration $\underset{\mathbb{B}}{\mathbb{P}}$ satisfies the induction principle w.r.t. $T$ if the functor T-Alg : $T$ - $\mathcal{A l g} \rightarrow \operatorname{Pred}(T)-\mathcal{A l g}$ preserves initial algebras, i.e. whenever $(D$, constr $)$ is an initial $T$-model, $1-\mathcal{A l g}(D$, constr $)$ is an initial $\operatorname{Pred}(T)$-model.

This definition means that for an object $P$ in $\mathbb{P}$, in order to give a morphism $f: T_{D} \rightarrow P$ it is sufficient to endow $P$ with a $\operatorname{Pred}(T)$-algebra, $(P, h: \operatorname{Pred}(T) P \rightarrow P)$. 
Note that if $P$ is a predicate over $D$, the condition is also necessary, as a morphism $f: T_{D} \rightarrow P$ gives a Pred $(T)$-algebra

$$
\operatorname{Pred}(T) P \stackrel{!}{\longrightarrow} T_{T D} \stackrel{T \text { (constr) }}{\longrightarrow} T_{D} \stackrel{f}{\longrightarrow} P
$$

For the more general case of the definition, the condition is also necessary if we assume $\mathbb{B}$ has image-factorisation for $T$-algebras, e.g. when $\mathbb{B}=$ Set.

We illustrate the logical import of the above definition with the polynomial functor of natural numbers and lists below. We assume the bicartesian structure of $\mathbb{P}$ is obtained as in Proposition 2.2.6. The internal language of $p$ in this case includes the logical connectives $\{\wedge, T, \vee, \perp\}$ and the coreindexing functors along coproduct injections. To simplify the presentation, we consider only the entailment relation $\vdash$ in the internal language, disregarding the proof terms. Note that for $\iota: I \rightarrow I+J$ in $\mathbb{B}$, given predicates $x: I \vdash Q(x)$ Prop and $y: I+J \vdash P(y)$ Prop a morphism $f: \iota !(Q) \rightarrow P$ corresponds under the adjunction $\iota ! \dashv \iota^{*}$ to a morphism $f^{\wedge}: Q \rightarrow \iota^{*}(P)$, which amounts to an entailment $x: I \mid Q(x) \vdash P(\iota x)$.

3.0.5. Examples. Let $\underset{\mathbb{B}}{\mathbb{P}}$ be as in Proposition 2.2.6.

(i) For the polynomial functor $T X=1+X$ in $\mathbb{B}$, the corresponding $\operatorname{Pred}(T)$ polynomial functor in $\mathbb{P}$ is $\operatorname{Pred}(T) H=\tilde{1} \tilde{+} H$. Let $P \in\left|\mathbb{P}_{I}\right|$ and let $(\mathrm{N},[z, s])$ be the initial $T$-model in $\mathbb{B}$. To give a $\operatorname{Pred}(T)$-algebra $(P, f: \operatorname{Pred}(T) P \rightarrow P)$ amounts to give a $T$ algebra $(I,[a, m]: T I \rightarrow I)$ (which induces a morphism it $(a, m): N \rightarrow I)$ and a vertical morphism $\hat{f}: \operatorname{Pred}(T) P \rightarrow[a, m]^{*}(P)$. Let us examine this vertical morphism in the internal language of $p$ : it corresponds to a sequent

$$
x: 1+I \mid \iota !\left(T_{1}\right) \vee \iota_{!}^{\prime}(P) \vdash P([a, m] x)
$$

which can be decomposed into two sequents

$$
x: 1+I\left|\iota !\left(T_{1}\right) \vdash P([a, m] x) \quad x: 1+I\right| \iota_{!}^{\prime}(P) \vdash P([a, m] x)
$$

which in turn correspond to sequents

$$
x^{\prime}: 1\left|T_{1} \vdash P(a) \quad y: I\right| P(y) \vdash P(m y)
$$

The above corresponds to the usual induction principle on the natural numbers: to prove $P(x)$ for the elements $x: I$ generated by $a$ and $m$, we must prove $P(a)$ and $P(y) \Longrightarrow P(m y)$. The validity of the induction principle in $p$ asserts then the existence (and uniqueness) of a morphism it $(f): T_{N} \rightarrow P$ over it $(a, m)$, which is the desired proof of the previously mentioned 'validity' of $P$ in the image of it $(a, m)$.

(ii) For the polynomial functor $T_{A} X=1+A \times X$, for some $A \in|\mathbb{B}|$, we get the polynomial functor $\operatorname{Pred}(T) Y=\tilde{1} \tilde{+} T_{A} \tilde{x} Y$. Let $(L,[$ nil, cons $])$ be the initial $T$-model and let $P \in\left|\mathbb{P}_{L}\right|$. Note that modulo the isomorphism [nil, cons] : $1+A \times L \rightarrow L$, the predicate $P$ corresponds to a predicate $P^{\prime}$ on $1+A \times L$, i.e. $x: 1+A \times L \vdash P^{\prime}(x)$. The predicate $P^{\prime}$ therefore determines two predicates $S$ and $Q$, with $x^{\prime}: 1 \vdash S \leftrightarrow P^{\prime}$ (nil) and $a: A, l: L \vdash Q(a, l) \leftrightarrow P^{\prime}(\operatorname{cons}(a, l))$. To give a vertical global element $h: T_{L} \rightarrow P$, a proof of the property $P$ for all lists, amounts to give a morphism $k: \operatorname{Pred}(T) P \rightarrow P$ over [nil, cons] : $1+A \times L \rightarrow L$. It corresponds to a sequent

$$
x: 1+A \times L \mid \iota !\left(T_{1}\right) \vee \iota_{!}^{\prime}\left(T_{A} \tilde{x} P\right) \vdash P^{\prime}([\text { nil, cons }] l)
$$


which can be decomposed into two sequents

$$
y: 1 \mid \vdash S
$$

and

$$
a: A, l: L \mid P(l) \vdash Q(a, l)
$$

where we have simplified the antecedent of the second sequent by $T_{A \times L} \wedge P(l) \rightarrow P(l)$. We thus get the usual structural induction principle for finite lists.

\section{Validity of the induction principle in the presence of compre- hension}

We now set out to show that, like in ordinary set theory, if the logic admits comprehension, the induction principle is valid in it. First, let us make an important remark.

4.0.6. REMARK. Since the structure map constr : $T D \rightarrow D$ of an initial algebra ( $D$, constr) is an isomorphism, cf. 2.1.3, it follows from Definition 3.0.4, that if $p$ satisfies the induction principle, the following condition must hold

$$
\operatorname{Pred}(T) T_{D} \stackrel{!}{\rightarrow} T_{T D} \text { is an isomorphism }
$$

Notice that the above morphism is the instance at $D$ of the 2 -cell $\rho: \operatorname{Pred}(T) T \Rightarrow T T$ in 3.0.3. Given that $T \in \mathcal{T}_{M}$, the condition that $\rho$ be an isomorphism amounts to requiring:

- For 0 , the initial object of $\mathbb{B}$, the initial object in $\mathbb{P}_{0}$ is terminal, that is, the fibre $\mathbb{P}_{0}$ is the terminal category 1 .

- For any pair of objects $I, J$ of $\mathbb{B},\left(\iota_{I, J}\right) !\left(T_{I}\right)+I+J\left(\iota_{I, J}^{\prime}\right) !\left(T_{I}\right) \cong T_{I+J}$

This last condition essentially means that the union of the images of the coproduct injections 'cover' the object $I+J$. We note that these conditions are satisfied for instance, when

- We consider internal logic fibrations, i.e. fibrations in which the predicates are subobjects of the base category, in which coproduct injections are monic.

- More generally, in the presence of comprehension, as $T$ preserves coproducts because it has a right adjoint.

- We consider the logic relative to a stable factorisation system, as in [Pav93], where predicates are interpreted as (equivalence classes of) formal monos.

From now on, we will assume condition (1) is satisfied. We recall from [Jac91] the definition of comprehension in a fibration (which is essentially the same as given in [Law70] for hyperdoctrines).

4.0.7. Definition. A fibration $\underset{\mathbb{P}}{\mathbb{P}}$ with a fibred terminal object $T: \mathbb{B} \rightarrow \mathbb{P}$ admits comprehension if $T$ has a right adjoint, $T \dashv\{-\}$. For an object $P$ over $X$, we write $\{X \mid P\}$ for the value of $\{-\}$ at $P$.

The above definition means that, given a morphism $f: Y \rightarrow X$ in $\mathbb{B}$ and a predicate $P \in\left|\mathbb{P}_{X}\right|, P(f)$ is provable iff the 'image' of $f$ lies in $\{X \mid P\}$. In Set comprehension is the usual operation $P \mapsto\{x \in X \mid P(x)\}$. Clearly, the fibrations of Examples 2.2.5 admit comprehension; in the second case, notice that an admissible subset of a cpo is itself a cpo. 
4.0.8. THEOREM. Let $\underset{\mathbb{P}}{\mathbb{P}}$ be a bicartesian fibration, which satisfies condition (1) and admits comprehension. Then, $p$ satisfies the induction principle w.r.t. every polynomial endofunctor on $\mathbb{B}$.

Proof. Condition (1) and $T \dashv\{-\}$ give data satisfying the hypothesis of Theorem 2.3.1. We then conclude that $T-\mathcal{A} l g$ has a right adjoint $\{-\}-\mathcal{A} l g$ and therefore preserves initial objects.

The import of the above theorem is that for an polynomial functor $T_{M}$, the functor $\{-\}$-Alg turns a Pred $(T)$-algebra on a predicate $P$ into a $T$-algebra on the 'extent' of the predicate $P$. This is the essential role comprehension plays in showing the validity of the induction principle in Set: given a predicate $P$ on the natural numbers $\omega$, which is inductive, we use the initiality of $\omega$ to conclude that the (inductive) subset $\{n \in \omega \mid P(n)\}$ must be the whole of $\omega$, and thus the predicate $P$ is (provably) true.

\section{Stability of initial algebras under weakening of context}

So far we have considered inductive data types and their associated induction principle in terms of initiality in the empty context. For instance, the initiality of $\mathrm{N}$ allows us to define functions out of it, e.g. $h: \mathrm{N} \rightarrow X$, by endowing the set $X$ with a $1+(-)$-algebra structure. But we also want to use this method when the inductive data type occurs in an arbitrary context, e.g. to define addition add : $\mathrm{N} \times \mathrm{N} \rightarrow \mathrm{N}$ by induction on the second argument. This requires that the initiality of $N$ be preserved when we move from the empty context to the context $n: \mathrm{N}$ (for the first argument of add). This operation is called context weakening. Technically, we say initiality is stable under addition of indeterminates, the indeterminate being $n: N$.

A similar extension is needed then for the associated induction principle, since when we perform context weakening $\Gamma \mapsto \Gamma, x: I$, the element $x$ may be subject to some (propositional) hypothesis. That is, we are generally interested in proving relative entailments $P \vdash Q$ rather than 'absolute' assertions $T \vdash Q$. For instance, we may want to prove $n: \mathrm{N}, m: \mathrm{N} \mid p: \operatorname{Even}(m) \vdash q: \operatorname{Even}(\operatorname{add}(2 * n, m))$ for some $q$, in which case we use induction on $n$ with $m: \mathrm{N}$ and $p: \operatorname{Even}(m)$ as parameters.

Abstractly, both extensions are instances of the same phenomenon: let $\mathcal{K}$ be a $2-$ category with finite products and inserters and let $A$ be an object of $\mathcal{K}$ with a 'terminal object' $! \dashv 1: 1 \rightarrow A$. Given any global element $I: \mathbf{1} \rightarrow \mathbf{A}$, we can consider the 'object $A$ with an indeterminate element $x: 1 \Rightarrow I$ ',$A[x: I]$. This object is equipped with $\eta_{I}: A \rightarrow A[x: I]$ and a 2-cell $\alpha_{x}: \eta_{I} 1 \Rightarrow \eta_{I} I$, and is universal among objects with such data. Given an endomorphism $T: A \rightarrow A$, we can consider the 'object of $T$-algebras' $T$-Alg, namely the inserter of $T$ and the identity on $A$. Similarly, since $T: A \rightarrow A$ induces $T[x: I]: A[x: I] \rightarrow A[x: I]$ with $T[x: I] \eta_{I}=\eta_{I} T$, we can consider the object $T[x: I]-\mathcal{A l g}$ and the induced morphism $\eta_{I}-\mathcal{A} l g: T-\mathcal{A l g} \rightarrow T[x: I]-\mathcal{A l g}$. Stability means that $\eta_{I}$-Alg preserves 'initial objects', for every $I: 1 \rightarrow A$. It follows from Theorem 2.3.1 that stability is guaranteed whenever the object $A$ is functionally complete, i.e. when $\eta_{I}$ has a right adjoint. We spell this out in more detail for categories and fibrations in the following subsections. Further details on indeterminates and functional completeness can be found in [HJ93]. We refer to [Str72] for the relevant definitions of comonads and their associated morphisms, as well as Kleisli objects for them in a 2-category. Anyway, these concepts are not essential to understand what follows. 


\subsection{Stability of initial algebras in a distributive bicategory}

The material in this subsection is based on [Jac95], although the formulations and proofs are different. It is just a preliminary to the treatment of stability of induction principles in $\S 5.2$.

Given a bicartesian category $\mathbb{B}$ and an object $I, \mathbb{B}[x: I]$ denotes the universal bicartesian category $\eta_{I}: \mathbb{B} \rightarrow \mathbb{B}[x: I]$ which has a global element of 'type' $I$, i.e. a morphism $x: 1 \rightarrow \eta_{I} I$. Universality means (at the 1-dimensional level) that given a bicartesian category $\mathbb{C}$, a functor $F: \mathbb{B} \rightarrow \mathbb{C}$ preserving finite products and coproducts, and a morphism $a: F 1 \rightarrow F I$, there is a unique functor $F^{\prime}: \mathbb{B}[x: I] \rightarrow \mathbb{C}$ preserving finite products and coproducts such that

$$
F^{\prime} \eta_{I}=F \quad F^{\prime}(x)=a
$$

The category $\mathbb{B}[x: I]$ can be characterised as the Kleisli category of the comonad (-) $\times I$, written $\mathbb{B} / / I$, when $\mathbb{B}$ is distributive, i.e. $-\times I$ preserves finite coproducts.

Logically, we think of $\mathbb{B}[x: I]$ as the theory with the same types of $\mathbb{B}$, whose terms have a 'parameter' of type $I$, i.e. they are terms of the form $\Gamma, x: I \vdash t: J$ in $\mathbb{B}$. This interpretation is obtained by considering the internal language of the Kleisli category of the comonad $-x I$ on $\mathbb{B}$.

A functor $T: \mathbb{B} \rightarrow \mathbb{B}$ lifts to a functor $T / / I: \mathbb{B} / / I \rightarrow \mathbb{B} / / I$ such that $(T / / I) \circ \eta_{I}=$ $\eta_{I} \circ T$, whenever it is endowed with the appropriate additional structure. Technically, this structure is exactly what makes $T$ a morphism of comonads; it is essentially the same as requiring $T$ strong, although this latter formulation leads to often misleading considerations of enrichement. More specifically, we require a natural transformation $(-\times I) T: \theta \Rightarrow T(-\times I)$ satisfying the following coherence conditions:

$$
F \pi_{J, I} \circ \theta_{J}=\pi_{F J, I} \quad \theta_{J \times I} \circ\left(\theta_{J, I} \times I\right) \circ\left\langle i d, \pi_{F J, I}^{\prime}\right\rangle=F\left\langle i d, \pi_{F J, I}^{\prime}\right\rangle \circ \theta_{J, I}
$$

for every object $J$ of $\mathbb{B}$. Every polynomial functor $T$ admits such structure and hence can be lifted to $\mathbb{B} / / I$.

5.1.1. Definition. Given a distributive category $\mathbb{B}$ and a polynomial functor $T: \mathbb{B} \rightarrow \mathbb{B}$, $\mathbb{B}$ admits stable initial $T$-algebras if it admits an initial $T$-algebra and for every object $I$, the functor $\left(\eta_{I}\right)-\mathcal{A l} g: T-\mathcal{A l} g \rightarrow(T / / I)-\mathcal{A l g}$ preserves initial objects.

We recall from [HJ93] that $\mathbb{B}$ is functionally complete if for every object $I$, the functor $\eta_{I}: \mathbb{B} \rightarrow \mathbb{B}[x: I]$ has a right adjoint. For $\mathbb{B}$ bicartesian this is the case precisely when it is (bi)cartesian closed. As an easy consequence of Theorem 2.3.1 we have the following result.

5.1.2. Proposition. Let $\mathbb{B}$ be a functionally complete distributive category (or equivalently, bicartesian closed). Whenever $\mathbb{B}$ has initial $T$-algebras, they are stable.

\subsection{Stability of initial algebras in a distributive fibration}

Just as we require inductive data types to be stable under addition of indeterminates to use its initial algebra property in an arbitrary context, we must require an analogous stability of their associated induction principles. In order to express such stability, we consider, for a given fibration (logic), an associated one with 'parameters' both on the base and total categories.

5.2.1. REMARK. Although the treatment of indeterminates for fibrations to follow parallels that for categories in $§ 5.1$, there is a subtle technical difference. All the concepts 
previously defined by universal properties in Cat, should be considered in their bicategorical variants in $\mathcal{F} i b$, i.e. up-to-equivalence rather than up-to-isomorphism. This is because the pseudo-functorial nature of the cleavages of fibrations allows only the existence of the bicategorical cocompleteness properties required (e.g. Kleisli objects), rather than the 2-categorical versions previously mentioned. The strict 2-categorical version does apply if we restrict attention to split fibrations and splitting-preserving morphisms.

Given a bicartesian fibration $\underset{\mathbb{B}}{\mathbb{P}}$ and an object $P$ of $\mathbb{P}$, the fibration with an indeterminate of $P$, written $p[\langle x, h\rangle: P]: \mathbb{P} / /(P) \rightarrow \mathbb{B}[x: p P]$ is the universal fibration $\left(\eta_{P}, \eta_{I}\right): p \rightarrow p[\langle x, h\rangle: P]$ with a global element $x: 1 \rightarrow \eta_{I}(I)$ in $\mathbb{B}[x: p P]$ and a global element $h: T_{1} \rightarrow x^{*}\left(\eta_{P}(P)\right)$ in $(\mathbb{P} / /(P))_{1}$. Universality means that given a bicartesian fibration $\underset{\mathbb{C}}{\mathbb{Q}} \underset{\mathrm{Q}}{\mathbb{Q}}$, a morphism $(H, K): p \rightarrow q$ preserving finite products and coproducts, and global elements $a: K 1 \rightarrow K(p P)$ and $b: H T_{1} \rightarrow a^{*}(H P)$, there is a unique (up to isomorphism) morphism $\left(H^{\prime}, K^{\prime}\right): p[\langle x, h\rangle: P] \rightarrow q$ preserving finite products and coproducts such that

$$
\left(H^{\prime}, K^{\prime}\right)\left(\eta_{P}, \eta_{I}\right) \cong(H, K) \quad K^{\prime} x=a \quad \phi \circ H^{\prime} h=b
$$

where $\phi: H^{\prime}\left(x^{*}\left(\eta_{P}(P)\right)\right) \rightarrow\left(K^{\prime \prime} x\right)^{*}(H P)$ is the canonical comparison isomorphism in the fibration $q$.

It is easy to extend Proposition 2.2.6 to make $\mathbb{P}$ a distributive category when the base and the fibres are so and when the coreindexing functors satisfy the Beck-Chevalley condition and Frobenius reciprocity, as formulated in [Law70]. We call such $p$ a distributive fibration. In this case, we can characterise $p[\langle x, h\rangle: P]$ as a Kleisli fibration $p / /(P)$ for the comonad $((-\tilde{x} P),(-\times p P))$ on $p$ (in $\mathcal{F} i b),[$ HJ93].

Logically, we think of the fibration $p[\langle x, h\rangle: P]$ as a logic with the same types and propositions as those of $p$, but whose terms have a 'parameter' of type $p P$, i.e. of the form $\Gamma, x: p P \vdash t: J$, and whose entailment relation allows an additional hypothesis $P(x)$, i.e. the entailments have the form

$$
\Gamma, x: p P \mid \Theta, h: P(x) \vdash q: Q(x)
$$

That is, we are assuming the presence of an additional element $x$ of type $I$, and a predicate of that type whose instance at $x$ is provably true. Both these elements represent the additional data with their associated properties forming the context in which we are working, for instance when carrying out an inductive proof. Semantically, such interpretation of $p[\langle x, h\rangle: P]$ can be obtained via the internal language of the Kleisli fibration of the comonad $((-\tilde{x} P),(-\times p P))$ on $p$.

A polynomial morphism $(\operatorname{Pred}(T), T): p \rightarrow p$ as considered in $\S 3$, induces an endomorphism $(\operatorname{Pred}(T)[h: P], T[x: p P]): p[\langle x, h\rangle: P] \rightarrow p[\langle x, h\rangle: P]$ such that

$$
(\operatorname{Pred}(T)[h: P], T[x: p P])\left(\eta_{P}, \eta_{I}\right) \cong\left(\eta_{P}, \eta_{I}\right)(\operatorname{Pred}(T), T)
$$

So we get a morphism $\left(\eta_{P}, \eta_{I}\right)-\mathcal{A} l g:(\operatorname{Pred}(T), T)-\mathcal{A l g} \rightarrow(\operatorname{Pred}(T)[h: P], T[x: p P])-\mathcal{A l g}$, where for an endomorphism in $\mathrm{Cat}^{\rightarrow}(H, K): p \rightarrow p$, with $p$ a fibration, $(H, K)-\mathcal{A l g}$ is the fibration obtained as the inserter of $(H, K)$ and the identity on $p$; its base category is $K-\mathcal{A l g}$ and its total one is $H-\mathcal{A l g}$. Now we can formulate stability of the induction principle for an inductive data type.

5.2.2. Definition. Given a polynomial functor $T: \mathbb{B} \rightarrow \mathbb{B}$, a distributive fibration $\underset{\mathbb{B}}{\mathbb{P}}$ satisfies the stable induction principle w.r.t. $T$ if $T$-Alg $: T$-Alg $\rightarrow \operatorname{Pred}(T)-\mathcal{A l g}$ preserves 
initial algebras and moreover, for every $P \in|\mathbb{P}|$, the morphism

$$
\left(\eta_{P}, \eta_{I}\right)-\mathcal{A l g}:(\operatorname{Pred}(T), T)-\mathcal{A} l g \rightarrow(\operatorname{Pred}(T)[h: P], T[x: p P])-\mathcal{A l g}
$$

preserves initial algebras (both on the base and the total categories).

5.2.3. REMARK. The above definition could equivalently be expressed by requiring that every fibration with an indeterminate $P, p[\langle x, h\rangle: P]$ satisfy the induction principle w.r.t. the induced morphism $(\operatorname{Pred}(T)[h: P], T[x: p P]): p[\langle x, h\rangle: P] \rightarrow p[\langle x, h\rangle: P]$, provided the base category $\mathbb{B}$ admits stable initial algebras. This makes logical sense, as we want to reason by induction in the fibration $p[\langle x, h\rangle: P]$, which has an indeterminate of type $p P$, satisfying the hypothesis $P$; this is exactly what the above formulation means.

In analogy to ordinary categories, we say that the fibration $\underset{\mathbb{B}}{\mathbb{P}}$ is functionally complete when, for every $P \in|\mathbb{P}|,\left(\eta_{P}, \eta_{I}\right): p \rightarrow p[\langle x, h\rangle: P]$ has a right adjoint (in $\mathcal{F}_{i} b$ ). This holds for instance when $p$ admits (or models) universal quantifiers $\forall$ and implication $\Longrightarrow$ (as a model of first-order logic). Then, we can apply Theorem 2.3.1 to show the following.

5.2.4. THEOREM. If $\eta_{P}: p \rightarrow p[\langle x, h\rangle: P]$ is a functionally complete distributive fibration and satisfies the induction principle w.r.t. to a polynomial endofunctor $T$, then it satisfies the stable induction principle w.r.t. to $T$.

The fibrations of Example 2.2.5 are functionally complete: $\begin{gathered}S u b(\mathcal{S e t}) \\ \text { Set } \\ \text { Sed }\end{gathered}$ is so because it models $\forall$ and $\Longrightarrow$, while $\begin{gathered}A S u b\left(c_{p o}\right) \\ \begin{array}{c}1 \text { cod } \\ \mathcal{C}_{p o} o\end{array}\end{gathered}$ is functionally complete although it does not model $\Longrightarrow$. Thus, the above abstract formulation seems to capture better this kind of example than a purely syntactic approach would. Functional completeness (at the logic level) is implicitly used in [LS86, §II.4] to show validity of induction over the natural numbers in a topos.

\section{Conclusions and further work}

Our aim was to give a precise abstract account of structural induction over data types, presenting the relevant technical machinery. A pay-off of this account is the precise relationship between logical predicates and induction. This relationship is further elucidated in a sequel to the present paper [HJ95], where we give an account of coinduction principles along the same lines as those for induction here. In that case, the 'equality predicate' functor takes over the role of $T$, and the fact that such functor preserves the relevant structure becomes (an instance of) Reynolds' 'identity extension lemma' [MR91]. There are also some considerations as to the extent the present approach can cope with bifunctoriality, in order to obtain (co)induction principles for recursive data types, in line with the domain-theoretic account in [Pit93].

We should mention that the approach here can be applied to formulate induction principles for data types with equational constraints (a standard kind of algebraic specification). The categorical aspects of such data types are described in [Jac95]. Briefly put, such data types are described by so-called distributive signatures $(\Sigma, E)$, and their models correspond to distributive functors $M: \mathcal{C}(\Sigma, E) \rightarrow \mathbb{B}$, where $\mathbb{B}$ is a distributive category and $\mathcal{C}(\Sigma, E)$ is the classifying category associated with the signature. A 'logical predicate' over such model is then a distributive functor $\operatorname{Pred}(M): \mathcal{C}(\Sigma, E) \rightarrow \mathbb{P}$ with 
$p \operatorname{Pred}(M)=M, c f .[$ Her93]. Induction can be stated by requiring that $T M: \mathcal{C} \ell(\Sigma, E) \rightarrow \mathbb{P}$ preserve initial models. This is the case when $p$ admits comprehension. Furthermore, in [Jac95] parametrized specifications correspond to morphisms $\phi:\left(\Sigma_{0}, E_{0}\right) \rightarrow(\Sigma, E)$, which semantically are interpreted as the functor sending a model $M: \mathcal{C l}\left(\Sigma_{0}, E_{0}\right) \rightarrow \mathbb{B}$ to the (distributive) left Kan extension along $\mathcal{C}(\phi): \mathcal{C}\left(\Sigma_{0}, E_{0}\right) \rightarrow \mathcal{C} \mathcal{C}(\Sigma, E)$. At the logical level, we expect a similar action on logical predicates as a suitable counterpart of induction for parametrized specifications. Once again, postcomposition with $T$ preserves (distributive) left Kan extensions in the presence of comprehension. This generalisation is a pay-off of the 2-categorical approach taken here. Of course, this topic requires further investigation to assess its suitability for applications in program development.

Further development of the ideas in this paper should account for some semantic features missing in the present treatment, notably partiality and type dependency.

\section{References}

[CS91] J.R.B. Cockett and D. Spencer. Strong categorical datatypes I. In Proceedings Category Theory 1991. Canadian Mathematical Society, 1991.

[Her93] C. Hermida. Fibrations, logical predicates and indeterminates. PhD thesis, University of Edinburgh, 1993. Tech. Report ECS-LFCS-93-277. Also available as Aarhus Univ. DAIMI Tech. Report PB-462.

[HJ93] C. Hermida and B. Jacobs. Fibrations with indeterminates: Contextual and functional completeness for polymorphic lambda calculi. In Book of A bstracts of Category Theory in Computer Science 5, september 1993. Extended version to appear in Mathematical Structures in Computer Science.

[HJ95] C. Hermida and B. Jacobs. Induction and coinduction via subset types and quotient types. presented at CLICS/TYPES workshop, Gōtenburg, january 1995.

[Jac91] B. Jacobs. Categorical Type Theory. PhD thesis, Nijmegen, 1991.

[Jac95] B. Jacobs. Parameters and parameterization in specification using distributive categories. Fundamenta Informaticae, to appear, 1995.

[Kel89] G.M. Kelly. Elementary observations on 2-categorical limits. Bulletin Australian Mathematical Society, 39:301-317, 1989.

[Law70] F.W. Lawvere. Equality in hyperdoctrines and comprehension scheme as an adjoint functor. In A. Heller, editor, Applications of Categorical Algebra. AMS Providence, 1970.

[LS81] D. Lehmann and M. Smyth. Algebraic specification of data types: A synthetic approach. Math. Systems Theory, 14:97-139, 1981.

[LS86] J. Lambek and P.J. Scott. Introduction to Higher-Order Categorical Logic, volume 7 of Cambridge Studies in Advanced Mathematics. Cambridge University Press, 1986.

[MR91] Q. Ma and J. C. Reynolds. Types, abstraction and parametric polymorphism 2. In S Brookes, editor, Math. Found. of Prog. Lang. Sem., volume 589 of Lecture Notes in Computer Science, pages 1-40. Springer Verlag, 1991.

[Pav90] D. Pavlović. Predicates and Fibrations. PhD thesis, University of Utrecht, 1990.

[Pav93] D. Pavlović. Maps I: relative to a factorisation system. Draft, Dept. of Math. and Stat., McGill University, 1993.

[Pit93] A. Pitts. Relational properties of recursively defined domains. Tech. Report TR321, Cambridge Computing Laboratory, 1993.

[Str72] R. Street. The formal theory of monads. Journal of Pure and Applied Algebra, 2:149$168,1972$.

[Str73] R. Street. Fibrations and Yoneda's lemma in a 2-category. In Category Seminar, volume 420 of Lecture Notes in Mathematics. Springer Verlag, 1973. 